\title{
碳气凝胶的孔结构及其对电化学超级电容器性能的影响
}

\author{
刘冬 ${ }^{1}$ 沈 军 ${ }^{1, *}$ 李亚捷 ${ }^{1}$ 刘念平 ${ }^{1}$ 刘 斌 ${ }^{2}$ \\ ('同济大学, 上海市特殊人工微结构材料与技术重点实验室, 上海 200092; \\ 2 航天特种材料及工艺技术研究所, 北京 7203 信箱)
}

\begin{abstract}
摘要：通过改变碳气凝胶的溶胶-凝胶制备条件和炭化活化工艺, 实现了对碳气凝胶纳米孔洞结构的控制. 采用 扫描电子显微镜(SEM)和氮气等温气体吸附法对碳气凝胶和 $\mathrm{KOH}$ 活化碳气凝胶的形貌和孔结构进行了表征和 分析, 并且使用循环伏安法(CV), 恒流充放电, 电化学阻抗谱(EIS)等检测技术评价了电化学性能. 结果表明: 发达 的三维纳米网络结构与合理的孔径分布是影响碳气凝胶电化学超级电容器性能的关键因素. 经适度活化后的碳 气凝胶材料含有丰富的介孔, 比表面积可达 $1480 \mathrm{~m}^{2} \cdot \mathrm{g}^{-1}$. 在 $6 \mathrm{~mol} \cdot \mathrm{L}^{-1}$ 的 $\mathrm{KOH}$ 溶液中, 在 $100 \mathrm{mV} \cdot \mathrm{s}^{-1}$ 的扫描速 率下其比电容量高达 $216 \mathrm{~F} \cdot \mathrm{g}^{-1}$. 通过拟合发现, 碳气凝胶类材料的大孔和介孔拥有更高的单位面积比电容量.
\end{abstract}

关键词: 碳气凝胶; 孔结构; 超级电容器; 电化学性能

中图分类号: 0646

\section{Pore Structures of Carbon Aerogels and Their Effects on Electrochemical Supercapacitor Performance}

\author{
LIU Dong ${ }^{1} \quad$ SHEN Jun ${ }^{1, *} \quad$ LI Ya-Jie ${ }^{1} \quad$ LIU Nian-Ping ${ }^{1} \quad$ LIU Bin $^{2}$ \\ ('Shanghai Key Laboratory of Special Microstructure Materials and Technology, Tongji University, Shanghai 200092, P. R. China; \\ ${ }^{2}$ Institute of Aerospace Special Materials and Technology, Beijing Mailbox 7203, P. R. China)
}

\begin{abstract}
Control of the pore structures of carbon aerogels (CAs) was investigated by changing the sol-gel polymerization and activation conditions. The morphologies and physical properties of the CAs and $\mathrm{KOH}$ activated carbon aerogels (ACAs) were characterized by scanning electron microscopy (SEM) and $\mathrm{N}_{2}$ adsorption isotherms. The electrochemical performances of the CAs and ACAs as electrode materials were characterized using cyclic voltammetry (CV), a galvanostatic charge-discharge test, and electrochemical impedance spectroscopy (EIS). The results showed that the well developed threedimensional nano-network structures and the reasonable pore size distributions of the CAs have great effect on their electrochemical performance in supercapacitors. Because of abundant mesopores and a high specific surface area $\left(1480 \mathrm{~m}^{2} \cdot \mathrm{g}^{-1}\right)$, the specific capacitance of a ACA electrode in $6 \mathrm{~mol} \cdot \mathrm{L}^{-1} \mathrm{KOH}$ electrolyte was approximately $216 \mathrm{~F} \cdot \mathrm{g}^{-1}$ at a scan rate of $100 \mathrm{mV} \cdot \mathrm{s}^{-1}$. A simple model was used to investigate the role of the pores in electrochemical performance.
\end{abstract}

Key Words: Carbon aerogel; Pore structure; Supercapacitor; Electrochemical performance

1 引 言

超级电容器是 20 世纪 60 年代发展起来的新型
储能器件, 是一种介于二次电池和传统物理电容之 间的新型储能器件. 它具有能量密度高、比功率高、

\footnotetext{
Received: November 23, 2011; Revised: January 16, 2012; Published on Web: February 17, 2012.

"Corresponding author. Email: shenjun67@tongji.edu.cn; Tel: +86-21-65986071.

The project was supported by the National Natural Science Foundation of China (11074189) and Shanghai Committee of Science and Technology, China (11nm0501600).

国家自然科学基金(11074189)和上海市科委纳米专项(11nm0501600) 资助项目
}

(C) Editorial office of Acta Physico-Chimica Sinica 
使用寿命长、充放电速度快、循环效率高等优点. 目 前, 按储能原理超级电容器电极材料可分为两类: 基于双电层原理的碳基材料; 基于法拉第赝电容效 应的金属氧化物、导电高聚物材料. ${ }^{1-5}$ 碳气凝胶是一 种三维纳米网络结构的多孔非晶碳素材料, 具有比 表面积高(活化后可以达到 $2500 \mathrm{~m}^{2} \cdot \mathrm{g}^{-1}$ 以上)、孔隙 率大(可达到 $2 \mathrm{~cm}^{3} \cdot \mathrm{g}^{-1}$ 以上)、孔径分布宽(0.3-100 $\mathrm{nm}$ )及导电性能好 (约 $\left.100 \mathrm{~S} \cdot \mathrm{cm}^{-1}\right)$ 等特点, 被认为是 一种理想的超级电容器电极材料. ${ }^{6-9}$

根据相关文献的报导, 材料的孔容、孔径分布、 比表面积、表面基团等是决定碳基材料比电容量的 主要因素. ${ }^{10-14} \mathrm{Lin}$ 等 ${ }^{11}$ 认为表面基团是决定碳材料 比电容量的重要因素. 他们比较了不同碳化温度得 到的碳气凝胶材料的表面基团, 结果表明: $600{ }^{\circ} \mathrm{C}$ 碳化得到的样品表面基团最丰富, 其比电容量也是 最高的. Wang 等 ${ }^{12}$ 通过对活化改性碳气凝胶的研究 认为比表面积和孔容决定碳材料比电容量, 并得出 了比电容量与比表面积和孔容成正比的结论. 而 $\mathrm{Shi}^{13}$ 认为孔径分布决定了活性炭材料的比电容量, 他把比表面积按微孔(孔径 $<2 \mathrm{~nm}$ ) 和介孔及大孔(孔 径 $>2 \mathrm{~nm}$ ) 分成了两类, 并计算得出介孔和大孔的单 位面积比电容量为 $(74 \pm 20) \mu \mathrm{F} \cdot \mathrm{cm}^{-2}$, 远远高于微孔

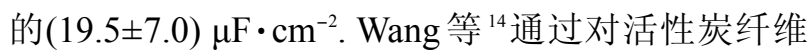
的研究也得出了与 Shi 相同的结果. 但他们对按孔 径大小把比表面积简单地区分为两类的处理方法 表示了质疑. 他们认为比表面积的电化学性质在一 个人为约定的数值 (孔径 $2 \mathrm{~nm}$ )上下有如此巨大的差 异是不符合物理学连续渐变的常识. 庄新国等 ${ }^{15}$ 使 用酚醛树脂基活性炭发现微孔比表面积的单位微 孔表面比电容为 $21.4 \mu \mathrm{F} \cdot \mathrm{cm}^{-2}$, 外孔表面比电容 $<10$ $\mu \mathrm{F} \cdot \mathrm{cm}^{-2}$. 但这一结果与 $\mathrm{Shi}^{13}$ 对通过活性炭微珠材料 的研究得出的结果完全不同, 庄新国等人认为不同 类型的碳材料其微观表面结构和表面基团差别很 大, 所以两者得出的结果不具有可比性.

从上述文献中我们可以看到, 碳材料的电化学 性能受多种因素影响. 碳材料的比电容量虽然在一 定条件下与比表面积成简单的正比关系, 但不同碳 材料得出的经验公式差异巨大, 不具有普遍性. 而 目前碳气凝胶材料的孔结构与电化学性能之间的 关系并没有得到充分的研究.

本文以间苯二酚和甲醛为原料, 碳酸钠为催化 剂, 通过改变溶胶-凝胶条件和 $\mathrm{KOH}$ 活化工艺, 实现 了对碳气凝胶材料孔结构的控制. ${ }^{16}$ 使用 SEM 和 $\mathrm{N}_{2}$
等温气体吸附对制备的材料进行了形貌和孔结构 分析; 使用循环伏安法 $(\mathrm{CV})$ 、恒流充放电、电化学阻 抗(EIS)等检测技术评价了电化学性能; 并探讨碳气 凝胶材料的比表面积、孔径分布与电化学性能之间 的关系.

\section{2 实 验}

\section{1 碳气凝胶的制备}

将间苯二酚 $(\mathrm{R}$, 分析纯)、甲醛( $\mathrm{F}$, 分析纯) 以 $1: 2$ 的摩尔比混合, 用适量碳酸钠 $(\mathrm{C}$, 分析纯)(均为国药 集团有限公司)作催化剂, 加入去离子水(GB668292 , 三级水)作为稀释剂用以调节反应物的质量组成 ( $M$ 为所有溶质占全部混合物的质量比), 室温下摚 拌 $15 \mathrm{~min}$ 使其均匀混合, 将混合液移至密闭容器 内, 放置恒温箱内 $\left((50 \pm 2){ }^{\circ} \mathrm{C}\right) 5 \mathrm{~d}$, 得到有机湿凝胶 $(\mathrm{RF})$. 将 $\mathrm{RF}$ 湿凝胶在丙酮(分析纯, 国药集团有限公 司)中浸泡 $5 \mathrm{~d}$, 每天更换一次, 然后放在空气中干燥 $3 \mathrm{~d}$, 得到 $\mathrm{RF}$ 气凝胶. 干燥后的 $\mathrm{RF}$ 气凝胶在 $\mathrm{N}_{2}$ 气氛 保护下, 以 $3^{\circ} \mathrm{C} \cdot \mathrm{min}^{-1}$ 的速率升温到 $1050{ }^{\circ} \mathrm{C}$ 并恒温 $3 \mathrm{~h}$, 使有机气凝胶均匀碳化为碳气凝胶 $(\mathrm{CAs})$, 最后 再降至室温. 本文中有机气凝胶的 $M$ 值为 $30 \%, \mathrm{R} / \mathrm{C}$ 摩尔比分别为 $500 、 1000 、 1500$ 和 2000 .

\section{$2.2 \mathrm{KOH}$ 活化碳气凝胶的制备}

将 $1 \mathrm{~g}$ 碳气凝胶与一定质量比的 $\mathrm{KOH}$ 固体在 20 $\mathrm{mL}$ 的陶瓷坩埚中均匀混合, 然后加入 $5 \mathrm{~mL}$ 无水乙醇 和 $10 \mathrm{~mL}$ 去离子水, $50^{\circ} \mathrm{C}$ 恒温搅拌 $1 \mathrm{~h}$. 把上述混合 物在 $120^{\circ} \mathrm{C}$ 恒温干燥 $12 \mathrm{~h}$, 然后放入管式炭化炉中, 在氮气气氛下 $\left(\right.$ 通气速率为 $200 \mathrm{~mL} \cdot \mathrm{min}^{-1}$ ) 以 $3^{\circ} \mathrm{C}$. $\mathrm{min}^{-1}$ 的升温速率加热到 $800^{\circ} \mathrm{C}$, 并恒温活化 $1 \mathrm{~h}$. 待 降到室温后取出样品, 然后浸入 $1 \mathrm{~mol} \cdot \mathrm{L}^{-1}$ 的盐酸溶 液中充分浸泡 $24 \mathrm{~h}$. 过滤后使用去离子水洗涤多次, 直至为中性, 然后将样品 $120^{\circ} \mathrm{C}$ 恒温干燥 $12 \mathrm{~h}$. 为了 方便记录, 我们采用 CAs-R/C-Kx 简写. 如 CAs$1500-\mathrm{K} 2$ 表示为 $\mathrm{R} / \mathrm{C}$ 摩尔比为 1500 , 与 2 倍质量的 $\mathrm{KOH}$ 活化后得到的活化碳气凝胶.

\section{3 测试与表征}

采用 Philips XL30 FEG 扫描电子显微镜(SEM) 分析样品的微观形貌, 加速电压为 $10 \mathrm{kV}$. 采用 Quantachrome Autosorb-1MP型全自动物理吸附 比表面分析仪测试液氮温度 $(77 \mathrm{~K})$ 下样品的 $\mathrm{N}_{2}$ 吸 附-脱附等温线, 并根据密度泛函理论( DFT)、HK (Horvath-Kawazoe)、t-plot 理论和 BJH (Barrett-JoynerHalenda)方法分析计算样品的比表面积、微/介孔孔 
径分布、微孔比表面积、微/介孔孔容.

\section{4 电化学性能测试}

将活性物质、乙炔黑和聚四氟乙烯(PTFE) $(60 \%)$ 以质量比 90:5:5 混合, 加入适量去离子水调成 糊状, 将上述物质均匀涂抹在泡沫镍集流体上, $105{ }^{\circ} \mathrm{C}$ 干燥 $12 \mathrm{~h}$, 用 $10 \mathrm{MPa}$ 的压力压 $1 \mathrm{~min}$, 最后裁 剪成 $1 \mathrm{~cm} \times 1 \mathrm{~cm}$ 的极片.

以氧化录电极作参比电极, 镍片做辅助电极, 采 用经典的三电极体系, 用上海辰华CHI660 A 电化学 工作站在 $6 \mathrm{~mol} \cdot \mathrm{L}^{-1} \mathrm{KOH}$ 中进行循环伏安、交流阻 抗、恒流充放电测试. 循环伏安测试扫描速率为 10-100 $\mathrm{mV} \cdot \mathrm{s}^{-1}$, 交流阻抗测试扫描频率为 $0.1-10000$ $\mathrm{Hz}$, 恒流充放电电流密度为 $1,5 、 10 \mathrm{~A} \cdot \mathrm{g}^{-1}$.

\section{3 结果与讨论}

\section{1 碳气凝胶的结构与形貌分析}

图 1 为 CAs-1500、CAs-1500-K4、CAs-1500-K6 和 CAs-500样品的 SEM 图. 从图 1(a)中可以看出, 碳 气凝胶材料具有无序三维纳米网络结构, 骨架网络 上的纳米碳颗粒成均匀球状. 从图 1(b,c)中可以看到 与图 1(a)类似的无序三维纳米网络结构, 并且纳米碳 颗粒之间的孔隙更多. 这表明经 $\mathrm{KOH}$ 活化后的碳气
凝胶材料不仅很好地保持了原有的骨架结构, 而且 可以打开一些原本封闭的纳米碳颗粒之间的孔隙.

图 2(a,b)为不同 $\mathrm{R} / \mathrm{C}$ 比的碳气凝胶和 $\mathrm{KOH}$ 活 化碳气凝胶的 $\mathrm{N}_{2}$ 吸脱附曲线, 从中可以看到碳气凝 胶材料的吸脱附曲线成 H3 型迟滞回线, 且在相对 高压力区没有表现出任何吸附限制, 表明碳气凝胶 材料是一种纳米颗粒组成的裂隙多孔碳材料, 含有 丰富的介孔和大孔. 图 2(c, d) 为不同碳气凝胶材料 的孔径分布图, 从图中可以看出碳气凝胶和 $\mathrm{KOH}$ 活 化碳气凝胶材料含 3 类孔: (A) 孔径小于 $2 \mathrm{~nm}$ 的微 孔; (B) 孔径在 2-4 nm 的小介孔; (C) 孔径大于 $4 \mathrm{~nm}$ 的大介孔和大孔. 碳气凝胶材料和 $\mathrm{KOH}$ 活化碳气凝 胶材料都具有大量 $A 、 C$ 两类孔和少量的 $B$ 类孔. 经 $\mathrm{KOH}$ 活化后的碳气凝胶含有更多的 $\mathrm{A} 、 \mathrm{~B}$ 类孔, 而 $\mathrm{C}$ 类孔含量基本不变. 这表明 $\mathrm{KOH}$ 活化能在不破坏碳 气凝胶材料原有的网络骨架结构的同时在碳纳米 颗粒上增加微孔和介孔含量.

表 1 为所制备的碳气凝胶和 $\mathrm{KOH}$ 活化碳气凝 胶的比表面积和孔结构参数. 从表 1 中可以看出, 碳 气凝胶材料的比表面积、微孔孔容与 $\mathrm{R} / \mathrm{C}$ 比成正向 关系, 同时微孔比表面积所占的比重也随 $\mathrm{R} / \mathrm{C}$ 比增 大而增加. 这是由于随着 $\mathrm{R} / \mathrm{C}$ 比的增大, 有机溶胶所
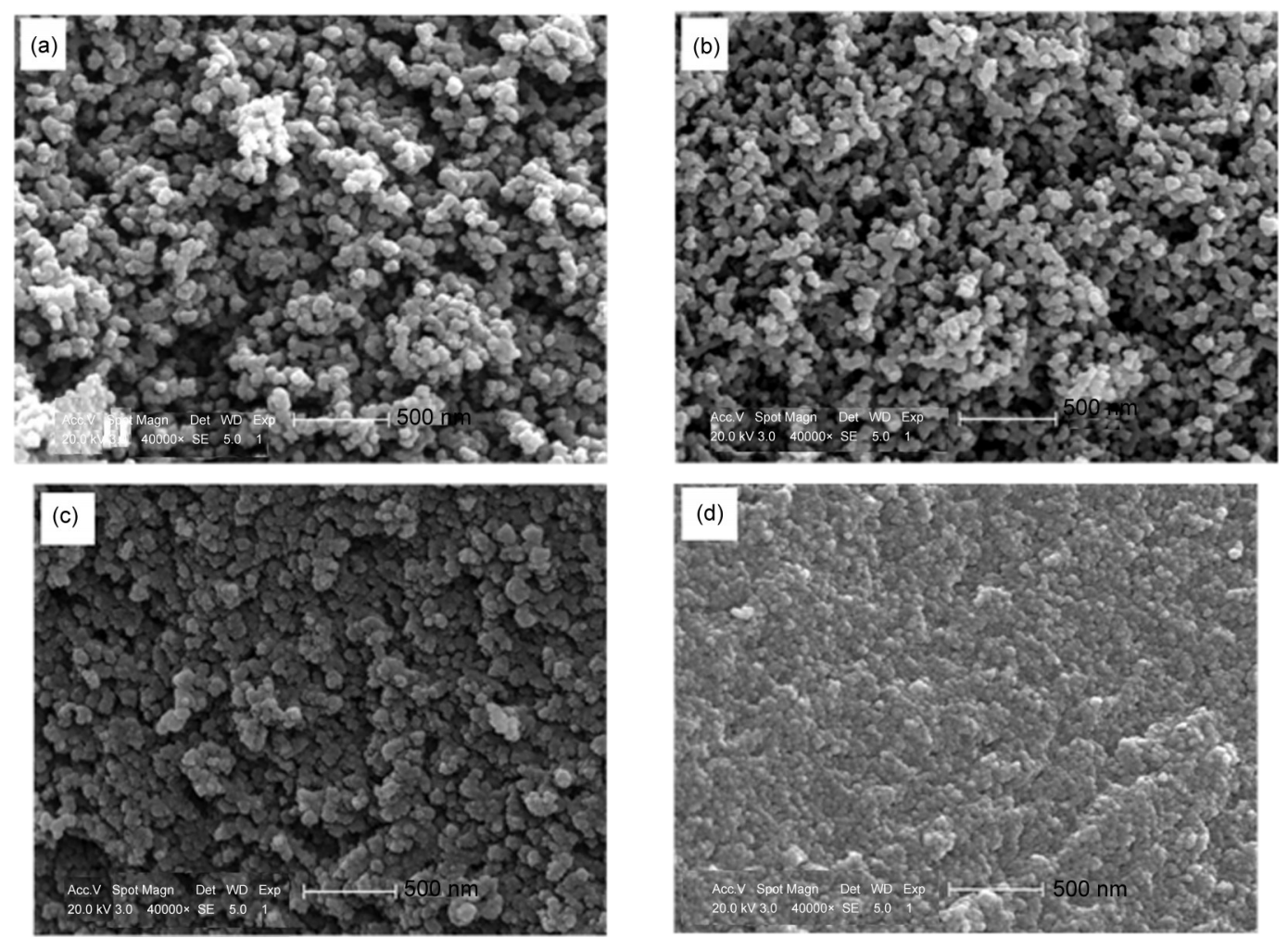

图 1 CAs-1500 (a)、CAs-1500-K4 (b)、CAs-1500-K6 (c) 和 CAs-500 (d) 样品的扫描电镜照片

Fig.1 SEM images of the samples CAs-1500 (a), CAs-1500-K4 (b), CAs-1500-K6 (c) and CAs-500 (d) CAs-R/C-Kx: CAs, carbon aerogels; $\mathrm{R} / \mathrm{C}$, the molar ratio of resorcinol to $\mathrm{Na}_{2} \mathrm{CO}_{3} ; x$, the mass ratio of $\mathrm{KOH}$ to carbon aerogels 

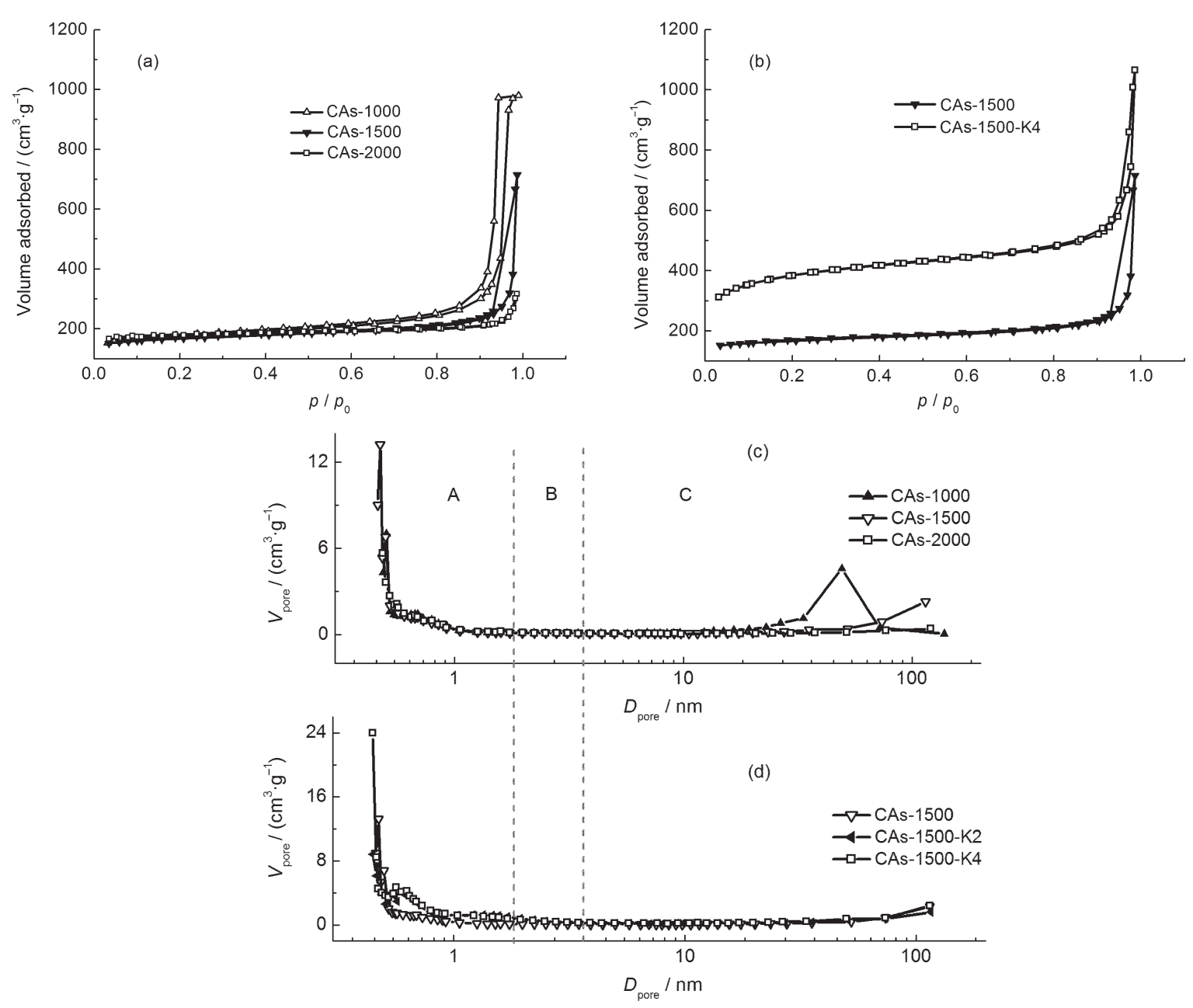

图 2 不同碳气凝胶样品的 $\mathbf{N}_{2}$ 吸脱附曲线和孔径分布

Fig.2 Nitrogen adsorption-desorption isotherms and pore size distribution of various samples A: pore size $<2 \mathrm{~nm}$; B: pore size $2-4 \mathrm{~nm}$; C: proe size $>4 \mathrm{~nm}$

需的凝胶时间越长, 有机气凝胶的骨架颗粒就越 大, 颗粒之间的连结更加的紧密, 从而减少了颗粒之 间的孔道比表面积. 从表 1 中可以看到, 经 $\mathrm{KOH}$ 活化 后碳气凝胶材料的比表面积、孔容都显著增加. 结合 图 2(d) 的分析可知, 适度的 $\mathrm{KOH}$ 活化不仅可以在纳 米碳颗粒上腐蚀出大量的 $\mathrm{A}$ 和 $\mathrm{B}$ 两类孔, 增加材料的 微孔和介孔比表面积, 而且还可以打开原本封闭的
纳米碳颗粒之间的孔隙, 增大材料的孔容. 同时发现 CAs-1500-K6 的微孔、介孔比表面积和孔容较 CAs1500-K4 都有所下降. 结合图 1(b, c) 的分析, 表明使 用过多的 $\mathrm{KOH}$ 的活化后碳气凝胶材料的骨架结构 经不住严重腐蚀而坡塌, 部分纳米碳颗粒之间孔隙 再次被封闭, 孔容减小, 比表面积也随之减少.

综合 SEM 形貌分析和氮气吸脱附曲线分析可

表 1 碳气凝胶及活化碳气凝胶的比表面积和孔结构参数

Table 1 Specific surface area and the pore structural parameters of CAs and ACAs

\begin{tabular}{lccccccc}
\hline \multicolumn{1}{c}{ Sample } & $S_{\text {DFT }} /\left(\mathrm{m}^{2} \cdot \mathrm{g}^{-1}\right)$ & $S_{\text {mic }} /\left(\mathrm{m}^{2} \cdot \mathrm{g}^{-1}\right)$ & $S_{\text {ex }} /\left(\mathrm{m}^{2} \cdot \mathrm{g}^{-1}\right)$ & $\left(S_{\text {ex }} / S_{\text {DFT }}\right) / \%$ & $V_{\mathrm{I}} /\left(\mathrm{cm}^{3} \cdot \mathrm{g}^{-1}\right)$ & $V_{\text {mic }} /\left(\mathrm{cm}^{3} \cdot \mathrm{g}^{-1}\right)$ & $D / \mathrm{nm}$ \\
\hline CAs-500 & 691 & 560 & 131 & 18.9 & 0.53 & 0.16 & 3.4 \\
CAs-1000 & 807 & 665 & 142 & 17.6 & 1.52 & 0.19 & 9.4 \\
CAs-1500 & 820 & 769 & 51 & 6.2 & 1.11 & 0.21 & 7.0 \\
CAs-2000 & 896 & 876 & 19 & 2.1 & 0.49 & 0.25 & 2.8 \\
CAs-1500-K2 & 1416 & 1310 & 106 & 7.5 & 1.40 & 0.49 & 3.9 \\
CAs-1500-K4 & 1480 & 1365 & 115 & 7.8 & 1.65 & 0.48 & 4.6 \\
CAs-1500-K6 & 1144 & 1045 & 99 & 8.6 & 1.56 & 0.35 & 5.9 \\
\hline
\end{tabular}

$S_{\mathrm{DFT}}$ : DFT specific surface area, $S_{\text {mic }}:$ micropore specific surface area, $S_{\text {ext }}$ external specific surface area, $V_{\mathrm{i}}$ : total volume, $V_{\text {mic }}$ : micropore volume, $D$ : average pore diameter 


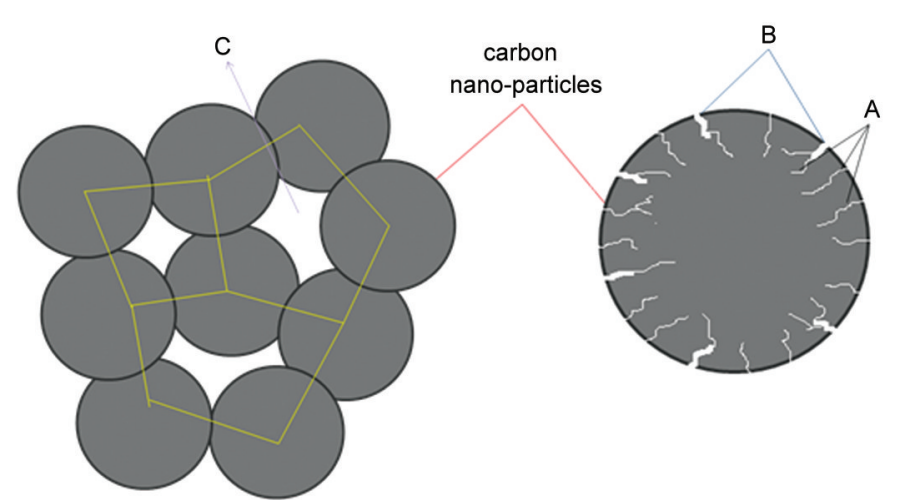

图 3 碳气凝胶材料孔结构的微观示意图

Fig.3 Schematic diagram of pore structures of CAs

以得到如图 3 所示的碳气凝胶材料的微观物理图 像. 我们认为 $\mathrm{A}$ 和 $\mathrm{B}$ 类孔是由碳气凝胶材料的纳米 碳颗粒上的裂隙和孔洞构成的, 而 C 类孔是由纳米 碳颗粒之间形成的网络孔道.

\section{2 孔结构与电化学性能分析}

\subsection{1 循环伏安特性}

图 4 为 CAs- 1500 和 CAs-1500-K4 在不同扫描 速率下的循环伏安曲线. 从图 4 中可以看到, 两种材 料的循环伏安曲线均成对称的矩形, 表现出典型的 电容器特性.

我们使用公式(1)计算各种材料的比电容量:

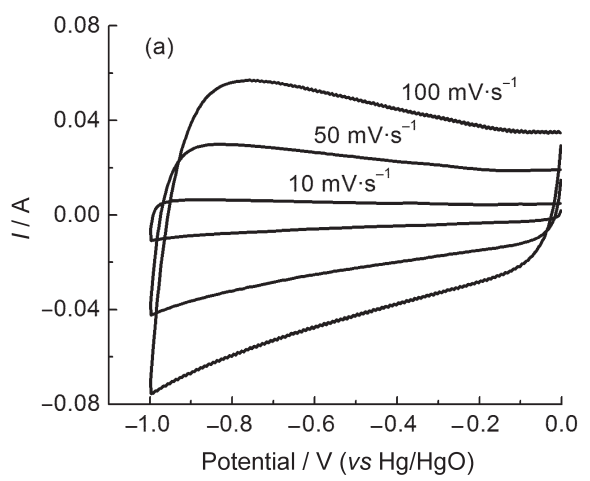

$$
C_{\mathrm{g}}=I /(m \cdot \Delta V)
$$

其中 $C_{\mathrm{g}}$ 为电极材料的比电容量, $I$ 为循环伏安扫描 的上半部分平台电流, $m$ 为电极活性物质质量, $\Delta V$ 为电压扫描速率. 计算得到的结果见表 2 . 从表 2 中 可以看到, 经 $\mathrm{KOH}$ 活化后碳气凝胶材料的比电容量 显著增加, CAs-1500-K4 在 $10 、 100 \mathrm{mV} \cdot \mathrm{s}^{-1}$ 的扫描速 率下其比电容量分别达到了 $229 、 216 \mathrm{~F} \cdot \mathrm{g}^{-1}$, 容量保 持率为 $94 \%$. 我们认为这是由于 $\mathrm{KOH}$ 活化在显著提 升碳气凝胶的微孔比表面积的同时, 能够打开纳米 碳微球之间的封闭孔隙, 降低了电解液离子在多孔 碳材料内的离子迁移电阻.

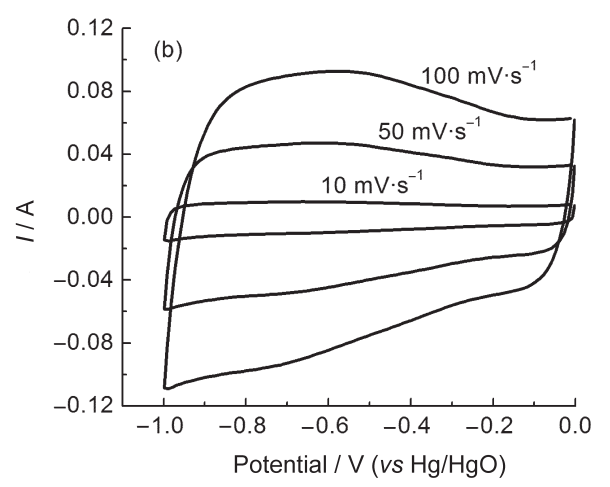

图 4 CAs-1500 (a)和 CAs-1500-K4 (b) 在不同扫描速率下的循环伏安曲线

Fig.4 Cyclic voltammograms of the samples CAs-1500 (a) and CAs-1500-K4 (b) at different scan rates

表 2 碳气凝胶及活化碳气凝胶的比电容

Table 2 Specific capacitance of the CAs and ACAs

\begin{tabular}{|c|c|c|c|c|c|}
\hline \multirow{2}{*}{ Sample } & \multirow{2}{*}{$S_{\mathrm{DFT}} /\left(\mathrm{m}^{2} \cdot \mathrm{g}^{-1}\right)$} & \multicolumn{3}{|c|}{ Specific capacitance $/\left(\mathrm{F} \cdot \mathrm{g}^{-1}\right)$} & \multirow{2}{*}{$\begin{array}{l}\text { Specific surface area } \\
\text { capacitance } /\left(\mu \mathrm{F} \cdot \mathrm{cm}^{-2}\right)\end{array}$} \\
\hline & & $10 \mathrm{mV} \cdot \mathrm{s}^{-1}$ & $50 \mathrm{mV} \cdot \mathrm{s}^{-1}$ & $100 \mathrm{mV} \cdot \mathrm{s}^{-1}$ & \\
\hline CAs-500 & 691 & 117 & 110 & 103 & 16.9 \\
\hline CAs- 1000 & 807 & 151 & 143 & 135 & 18.6 \\
\hline CAs- 1500 & 820 & 151 & 139 & 133 & 18.4 \\
\hline CAs-2000 & 896 & 110 & 96 & 89 & 12.3 \\
\hline CAs- $1500-\mathrm{K} 2$ & 1416 & 219 & 215 & 210 & 15.5 \\
\hline CAs-1500-K4 & 1480 & 229 & 218 & 216 & 15.5 \\
\hline CAs-1500-K6 & 1144 & 216 & 205 & 195 & 18.9 \\
\hline
\end{tabular}

Specific surface area capacitance $=$ Specific capacitance $\left(10 \mathrm{mV} \cdot \mathrm{s}^{-1}\right) / S_{\mathrm{DFT}}$ 
对于基于双电层效应的碳基材料, 其比电容量可 以用经典宏观的平行平面电容公式(2)做简单计算: ${ }^{18}$

$$
C_{\mathrm{g}}=\varepsilon \cdot S_{\mathrm{g}} / d
$$

其中 $\varepsilon$ 为电解液的介电常数, $S_{\mathrm{g}}$ 为电极材料的比表面 积, $d$ 为电解液离子与电极材料表面的距离. 从公式 (2)中可以看出, 碳基材料的比电容量应与材料的比 表面积呈线性关系. 但从表 2和图 5(a)可以看到, 碳 气凝胶材料的比电容量没有简单随着比表面积的 增加而变大, 更有 CAs-2000 比 CAs-1000 高出了 88 $\mathrm{m}^{2} \cdot \mathrm{g}^{-1}$ 的比表面积, 但其比电容量却下降了 $40 \mathrm{~F}$. $\mathrm{g}^{-1}$. 对于这种现象, 我们使用 $\mathrm{Shi}^{13}$ 的方法, 将材料的 比表面积分成两类, 即 $S_{\mathrm{mic}}$ (内孔小于 $2 \mathrm{~nm}$ ) 和 $S_{\mathrm{ext}}$ (外 通道孔大于 $2 \mathrm{~nm}$ ), 相对应的单位面积比电容量为 $C_{\text {mic }}$ 和 $C_{\text {ext. }}$. 电极的比电容量可用公式(3)计算:

$$
C_{\mathrm{g}}=S_{\mathrm{mic}} \cdot C_{\mathrm{mic}}+S_{\mathrm{ext}} \cdot C_{\text {ext }}
$$

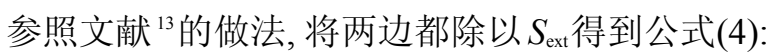

$$
C_{\mathrm{g}} / S_{\mathrm{ext}}=\left(S_{\mathrm{mic}} / S_{\mathrm{ext}}\right) \cdot C_{\mathrm{mic}}+C_{\mathrm{ext}}
$$

做 $C_{\mathrm{g}} / S_{\mathrm{ext}}$ 和 $S_{\mathrm{mic}} / S_{\mathrm{ext}}$ 的线性曲线拟合, 就可得到 $C_{\mathrm{mic}}$ 和 $C_{\text {ext }}$ 的值. 考虑到微孔的作用, 我们使用 $10 \mathrm{mV} \cdot \mathrm{s}^{-1}$ 的
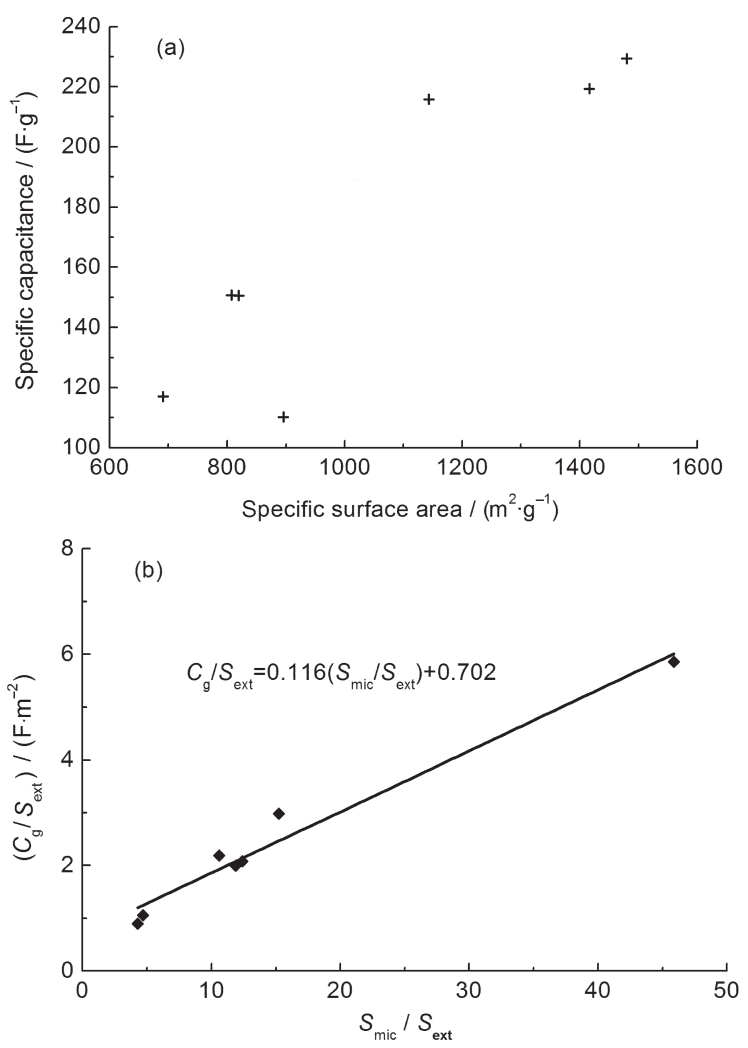

图 5 碳气凝胶材料的比电容量与 DFT 比表面积(a)及 $C_{\mathrm{g}} / S_{\text {ext }}$ 和 $S_{\text {mic }} / S_{\text {ext }}$ 线性拟合曲线(b)

Fig.5 Specific double layer capacitances vs total DFT surface areas for all CAs (a) and $C_{\mathrm{g}} / S_{\mathrm{ext}}$ plot as a function of $S_{\text {mic }} / S_{\text {ext }}$ for all CAs (b)
数据进行拟合, 得到图 5(b) 和公式(5):

$$
C_{\mathrm{g}} / S_{\text {ext }}=0.116 \cdot\left(S_{\text {mic }} / S_{\text {ext }}\right)+0.702
$$

计算得到 $C_{\text {mic }}$ 和 $C_{\text {ext }}$ 分别为 $(11.6 \pm 0.9) \mu \mathrm{F} \cdot \mathrm{cm}^{-2}$ 和 $(70.2 \pm 18.0) \mu \mathrm{F} \cdot \mathrm{cm}^{-2}$, 线性相关系数为 0.96 . 这一结 果与 Shi 使用活性碳颗粒材料报道的结果一致, 说 明单位面积比电容量的分段处理在碳材料中具有 一定的普适性. 但为什么单位面积比电容量在 $2 \mathrm{~nm}$ 这样一个人为约定的数字上下有如此大的差异, 其 中的物理化学过程目前并没有得到完备的研究. ${ }^{18-21}$ 通过以上研究可知, 大孔和介孔对碳基双电层电容 器是至关重要的, 他们不仅拥有更高的单位面积比 电容量, 而且在快速扫描下的表现更加优异.

\section{2 .2 恒流充放电测试}

图 6 为 CAs-1500-K4 在不同电流密度下的恒流 充放电曲线. 从图 6 可以看到曲线都成对称三角形, 表明 CAs-1500-K4 具有很高的充放电效率. 另外, 放 电开始的瞬间, 电压突降很小, 表明电极中活性物质 与电解液的接触良好, 电阻很小, 用公式(6)计算, 电 极内阻在 $1 \mathrm{~A} \cdot \mathrm{g}^{-1}$ 的电流密度下仅为 $0.4 \Omega$.

$$
R=\Delta U / I
$$

其中 $\Delta U$ 为电压突降, $I$ 为充放电电流.

\subsection{3 交流阻抗测试}

图 7(a) 为碳气凝胶及 $\mathrm{KOH}$ 活化碳气凝胶的 Nyquist 图. 从图中可以看到碳气凝胶和 $\mathrm{KOH}$ 活化 碳气凝胶具有相似的图谱: 高频段成半圆弧状, 较 高频区成 $45^{\circ}$ 斜线, 低频区为坚直向上的直线. ${ }^{2}$ 高频 段为电极材料的电阻阻抗特性, 从图中可得到 CAs-1500、CAs-1500-K4 和 CAs-1500-K6 的高频段 阻抗 $\left(R_{\mathrm{L}}\right)$ 分别为 $0.16 、 0.14$ 和 $0.15 \Omega$, 表明碳气凝胶 及 $\mathrm{KOH}$ 活化碳气凝胶材料具有良好的导电性. 较高 频段的 $45^{\circ}$ 斜线表现了电解液离子在多孔碳电极中

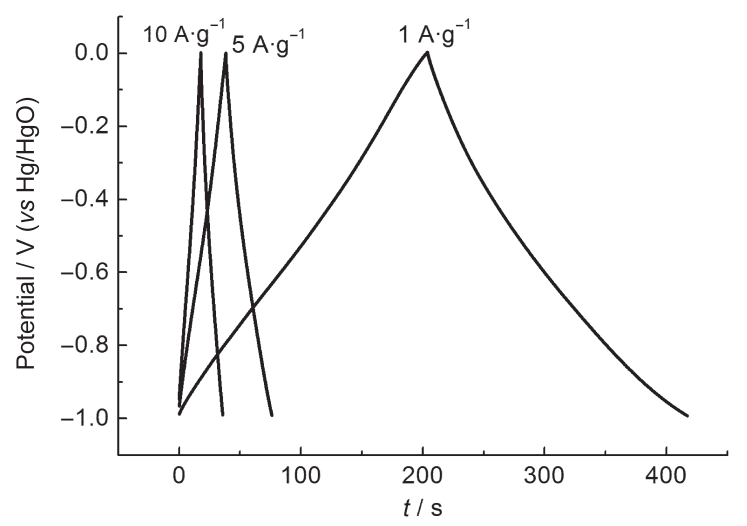

图 6 CAs-1500-K4 在不同电流密度下的恒流充放电曲线

Fig.6 Charge-discharge curves of CAs-1500-K4 at different constant current densities 

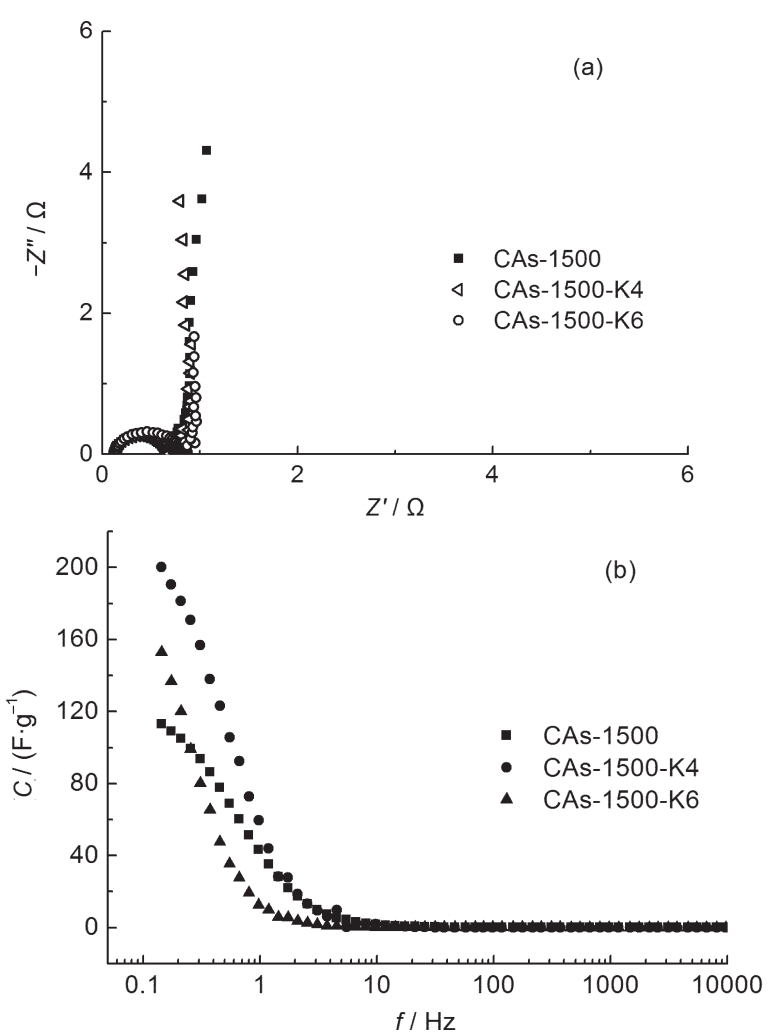

图 7 碳气凝胶电极交流阻抗图谱

Fig.7 AC impedance spectra of CAs

(a) Nyquist plots of EDLCs, (b) capacitance $v$ f frequency. EDLCs: electrical double-layer capacitors

扩散的 Warburg 阻抗特性. 低频区的坚直直线是由 电荷饱和控制, 表明电极具有良好的电容行为特性.

图 7(b) 为碳气凝胶及 $\mathrm{KOH}$ 活化碳气凝胶的电 容-频率响应曲线. 从图中可以看到 CAs-1500-K4 拥有最佳的电容一频率响应, 这是由于 $\mathrm{KOH}$ 活化增 加了材料的 $\mathrm{B} 、 \mathrm{C}$ 类孔, 降低了电解离子在电极中的 迁移电阻. CAs-1500-K6 在低于 $0.25 \mathrm{~Hz}$ 的区间内比 CAs-1500 表现出更佳的电容-频率响应特性, 这是 由于过度的 $\mathrm{KOH}$ 活化只增加材料的微孔, 而这些微 孔在高频段具有较大的离子迁移电阻, 所以无法显 现出电容特性.

\section{4 结 论}

(1) 碳气凝胶及 $\mathrm{KOH}$ 活化碳气凝胶材料的孔洞 是由纳米碳微球上的微孔、小介孔及纳米碳微球之 间的大介孔和大孔组成, 并由此作出了碳气凝胶类 材料的微观结构示意图.

(2) 通过循环伏安、恒流充放电、电化学交流阻抗 等测试表明, $\mathrm{KOH}$ 活化碳气凝胶材料具有优异的电 化学性能, 其比电容量在 $100 \mathrm{mV} \cdot \mathrm{s}^{-1}$ 的扫描速率下达 到 $216 \mathrm{~F} \cdot \mathrm{g}^{-1}$.
(3) 通过拟合得到了微孔、介孔和大孔的单位面 积比电容量, 结果表明微孔的单位面积比电容量小 于介孔和大孔的.

\section{References}

(1) Frackowiak, E.; Beguin, F. Carbon 2001, 39, 937.

(2) Conway, B. E. Electrochemical Supercapacitors Scientific Fundamentals and Technological Applications; Kluwer Academic/Plenum Publishers: New York, 1999.

(3) Lu, X. J.; Dou, H.; Yang, S. D.; Hao, L.; Zhang, F.; Zhang, X. G. Acta Phys. -Chim. Sin. 2011, 27, 2333. [卢向军, 窦 辉, 杨苏东, 郝 亮, 张 方, 张校刚. 物理化学学报, 2011, 27, 2333.]

(4) Xue, R.; Yan, J. W.; Tian, Y.; Yi, B. L. Acta Phys. -Chim. Sin. 2011, 27, 2340. [薛荣, 阎景旺, 田 颖, 衣宝廉. 物理化学 学报, 2011, 27, 2340.]

(5) Cai, J. J.; Kong, L. B.; Zhang, J.; Luo, Y. C.; Kang, L. Chin. Chem. Lett. 2010, 21, 1509.

(6) Pekala, R. W.; Farmer, J. C.; Alviso, C. T.; Tran, T. D.; Mayer, S. T.; Miller, J. M.; Dunn, B. J. Non-Cryst. Solids 1998, 225, 74.

(7) Li, J.; Wang, X. Y.; Wang, Y.; Huang, Q. H.; Dai, C. L.; Gamboa, S.; Sebastian, P. J. J. Non-Cryst. Solids 2008, 354, 19.

(8) Saliger, R.; Fischer, U.; Herta, C.; Fricke, J. J. Non-Cryst. Solids 1998, 225, 81 .

(9) Xu, Z. J.; Ji, T.; Zhao, L.; Wang, W. Y.; Yang, C. Y.; Gan, L. H. Acta Phys. -Chim. Sin. 2012, 28, 361. [徐子颉, 吉 涛, 赵 蕾, 王玮衍, 杨春艳, 甘礼华. 物理化学学报, 2012, 28, 361.]

(10) Liu, Y. F.; Hu, Z. H.; Xu, K.; Zheng, X. W.; Gao, Q. Acta Phys. -Chim. Sin. 2008, 24, 1143. [刘亚菲, 胡中华, 许 琨, 郑祥伟, 高 强. 物理化学学报, 2008, 24, 1143.]

(11) Lin, C.; Ritter, J. A.; Popov, B. N. J. Electrochem. Soc. 1999, $146,3639$.

(12) Wang, J. B.; Yang, X. Q.; Wu, D. C.; Fu, R. W.; Dresselhausc, M. S.; Dresselhausc, G. J. Power Sources 2008, 185, 589.

(13) Shi, H. Electrochim. Acta 1996, 41, 1633.

(14) Wang, J.; Chen, M. M.; Wang, C. Y.; Wang, J. Z.; Zheng, J. M. J. Power Sources 2011, 196, 550.

(15) Zhuang, X. G.; Yang, Y. S.; Ji, Y. J.; Yang, D. P.; Tang, Z. Y. Acta Phys. -Chim. Sin. 2003, 19, 689. [庄新国, 杨裕生, 嵇友菊, 杨冬平, 唐致远. 物理化学学报, 2003, 19, 689.]

(16) Aegerter, M. A.; Leventis, N.; Koebel, M. M. Aerogels Handbook; Springer: New York, 2011; pp 813-826.

(17) Pekala, R. W. J. Mater. Sci. 1989, 24, 3221.

(18) Chmiola, J.; Yushin, G.; Gogotsi, Y.; Portet, C.; Simon, P.; Taberna, P. L. Science 2006, 313, 1760.

(19) Kiyohara, K. J.; Sugino, T. S.; Asaka, K. J. J. Chem. Phys. 2010, 132, 144705.

(20) Barbieri, O.; Hahn, M.; Herzog, A. Carbon 2005, 43, 1303.

(21) Largeot, C.; Portet, C.; Chmiola, J.; Taberna, P. L.; Gogotsi, Y.; Simon, P. J. Am. Chem. Soc. 2008, 130, 2730. 